\title{
Vertex disjoint 4-cycles in bipartite tournaments
}

\author{
C. Balbuena ${ }^{\mathrm{a}, \mathrm{b}, *}$, D. González-Moreno ${ }^{\mathrm{a}}$, M. Olsen ${ }^{\mathrm{a}}$ \\ a Departamento de Matemáticas Aplicadas y Sistemas, Universidad Autónoma Metropolitana Unidad Cuajimalpa, México D.F., Mexico \\ b Departament de Matemàtica Aplicada III, Universitat Politècnica de Catalunya, Barcelona, España, Spain
}

\section{A R T I C L E I N F O}

\section{Article history:}

Received 22 May 2015

Received in revised form 22 October 2017

Accepted 23 October 2017

Available online $\mathrm{xxxx}$

\section{Keywords:}

Bipartite tournament

Vertex-disjoint cycles

Prescribed length

Minimum outdegree

Bermond-Thomassen conjecture

\begin{abstract}
A B S T R A C T
Let $k \geq 2$ be an integer. Bermond and Thomassen conjectured that every digraph with minimum out-degree at least $2 k-1$ contains $k$ vertex-disjoint cycles. Recently Bai, Li and Li proved this conjecture for bipartite digraphs. In this paper we prove that every bipartite tournament with minimum out-degree at least $2 k-2$, minimum in-degree at least 1 and partite sets of cardinality at least $2 k$ contains $k$ vertex-disjoint 4 -cycles whenever $k \geq 3$. Finally, we show that every bipartite tournament with minimum degree $\delta=\min \left\{\delta^{+}, \delta^{-}\right\}$ at least $1.5 k-1$ contains at least $k$ vertex-disjoint 4 -cycles.
\end{abstract}

(C) 2017 Published by Elsevier B.V.

\section{Introduction and terminology}

Bermond and Thomassen [5] posted the following conjecture, which relates the number of disjoint cycles in a digraph with the minimum out-degree.

Conjecture $1.1([5])$. Every digraph $D$ with $\delta^{+}(D) \geq 2 k-1$ has $k$ disjoint cycles.

This conjecture has been proved for general digraphs when $k=2, k=3$ and for tournaments [3,6,7,8]. Thomassen [8] established the existence of a finite integer $f(k)$ such that every digraph of minimum out-degree at least $f(k)$ contains $k$ disjoint cycles. Alon [1] proved in 1996 that for every integer $k$, the value $64 k$ is suitable for $f(k)$.

A bipartite tournament is an oriented complete bipartite graph. Observe that, the girth of any bipartite tournament containing a cycle is four. We denote a cycle of length four by $C_{4}$. Very recently, Bay, Li and Li [2], proved Conjecture 1.1 for bipartite tournaments as a consequence of another result related to the numbers of vertex disjoint cycles of a given length in bipartite tournaments with minimum out-degree at least $q r-1$, for $q \geq 2$ and $r \geq 1$ two integers. In this paper we will only consider bipartite tournaments. First, we present an alternative proof of this conjecture in a direct way for bipartite tournaments. We also prove that every bipartite tournament with minimum out-degree at least $2 k-2$, minimum in-degree at least 1 and partite sets of cardinality at least $2 k$ contains $k$ disjoint 4-cycles whenever $k \geq 3$. Finally, we show that every bipartite tournament with both minimum out-degree and minimum in-degree at least $(3 k-1) / 2$, contains at least $k$ disjoint cycles for all $k \geq 2$.

For terminology and notation we follow the book by Bang-Jensen and Gutin [4]. Through this work only finite digraphs without loops and multiple edges are considered. Let $D$ be a digraph with vertex set $V(D)$ and arc set $A(D)$. Two subdigraphs $D_{1}$ and $D_{2}$ of $D$ are disjoint if their vertex sets are disjoint. We denote by $\delta^{+}(D)$ the minimum out-degree of a vertex in $D$, by

\footnotetext{
* Corresponding author at: Departament de Matemàtica Aplicada III, Universitat Politècnica de Catalunya, Barcelona, España, Spain.

E-mail addresses: m.camino.balbuena@upc.edu (C. Balbuena),dgonzalez@correo.cua.uam.mx (D. González-Moreno), olsen@correo.cua.uam.mx
} (M. Olsen). 


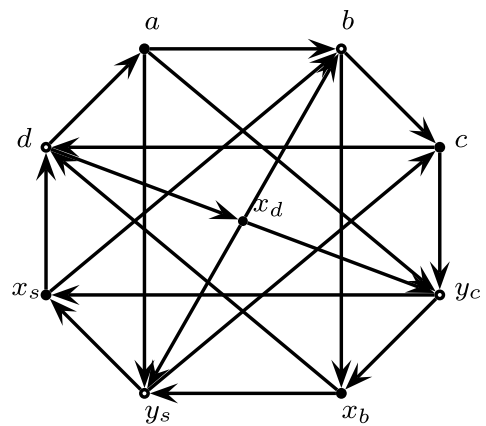

Fig. 1. Bipartite tournament with $\delta^{+}=2$ and $\delta^{-}=1$ without two disjoint 4-cycles

$\delta^{-}(D)$ the minimum in-degree of a vertex in $D$, and by $\delta(D)=\min \left\{\delta^{+}(D), \delta^{-}(D)\right\}$ the minimum degree of $D$. Two vertices $u$ and $v$ are twins if $N^{+}(u)=N^{+}(v)$ and $N^{-}(u)=N^{-}(v)$. A vertex $v$ with $d^{-}(v)=0$ is called a source. Similarly, a vertex $v$ with $d^{+}(v)=0$ is called a sink. The minimum length of a cycle in $D$ is called the girth of $D$. For a set $X \subseteq V(D)$, we use the notation $D[X]$ to denote the subdigraph of $D$ induced by the vertices of $X$. Let $u v$ be an arc of $D$. By reversing the arc $u v$, we mean that we replace the arc $u v$ by the arc $v u$. The converse of a digraph $D$ is the digraph $H$ obtained from $D$ by reversing all arcs.

\subsection{Results}

Conjecture 1.1 is proved for bipartite tournaments in [2]. In Theorem 1.1 we present an alternative proof of this result in a direct and short way. Our proof is the starting point for obtaining the rest of results contained in this paper.

Theorem 1.1. Let $k \geq 2$ be an integer. If $T$ is a bipartite tournament with $\delta^{+}(T) \geq 2 k-1\left(\operatorname{or}^{-}(T) \geq 2 k-1\right)$, then $T$ has at least $k$ disjoint cycles.

By Theorem 1.1, Conjecture 1.1 holds for bipartite tournaments. Next we give sufficient conditions to prove that every bipartite tournament with minimum out-degree at least $2 k-2$, minimum in-degree at least 1 and partite sets of cardinality at least $2 k$ contains $k$ disjoint 4 -cycles whenever $k \geq 3$.

Theorem 1.2. Let $k \geq 3$ be an integer. If $T$ is a bipartite tournament with $\delta^{+}(T) \geq 2 k-2, \delta^{-}(T) \geq 1$ and partite sets of cardinality at least $2 k$, then $T$ has at least $k$ disjoint cycles.

Remark 1.1. The following bipartite tournament (for $k=2$ ) with $\delta^{+}=2, \delta^{-}=1$, and partite sets of cardinality at least 4 , has no two disjoint $C_{4}$, see Fig. 1. Hence, the condition $k=3$ is necessary in Theorem 1.2.

Let $T$ be the bipartite tournament with partite sets $X=\left\{a, c, x_{b}, x_{s}, x_{d}\right\}$ and $Y=\left\{b, d, y_{s}, y_{c}\right\}$. The arcs of $T$ are the following:

$$
\begin{aligned}
& N^{+}(a)=\left\{b, y_{s}, y_{c}\right\}=N^{+}\left(x_{d}\right), N^{-}(a)=\{d\}=N^{-}\left(x_{d}\right) ; \\
& N^{+}(c)=\left\{d, y_{c}\right\}, N^{-}(c)=\left\{b, y_{s}\right\} ; \\
& N^{+}\left(x_{s}\right)=\{b, d\}, N^{-}\left(x_{s}\right)=\left\{y_{s}, y_{c}\right\} ; \\
& N^{+}\left(x_{b}\right)=\left\{d, y_{s}\right\}, N^{-}\left(x_{b}\right)=\left\{b, y_{c}\right\} .
\end{aligned}
$$

Considering digraphs with a given girth, Bang-Jensen, Bessy and Thomassé [3] established the following conjecture.

Conjecture 1.2 ([3]). Every digraph $D$ with girth $g \geq 2$ and minimum degree $\delta^{+}(D) \geq \frac{g}{g-1} k$ contains $k$ disjoint cycles.

Clearly, in order to a bipartite digraph has $k$ disjoint cycles it must have partite sets of cardinality at least $2 k$. Hence, Conjecture 1.2 can be easily corrected adding this requirement on the cardinality of the partite sets.

Corollary 1.1. Conjecture 1.2 holds for bipartite tournaments with $\delta^{-}(D) \geq 1$ and partite sets of cardinality at least $2 k$ for $k=2,3,4$.

Theorem 1.3. Every bipartite tournament with $\min \left\{\delta^{+}, \delta^{-}\right\} \geq 2$ has at least 2 disjoint cycles.

Remark 1.2. Theorem 1.3, is best possible as shown for the bipartite tournament described in Remark 1.1, see Fig. 1.

Finally, we establish that if both the minimum out-degree and minimum in-degree are at least $(3 k-1) / 2$, then the bipartite tournament has at least $k$ disjoint $C_{4}$.

Theorem 1.4. Let $k \geq 2$ be an integer. If $T$ is a bipartite tournament such that $\min \left\{\delta^{+}, \delta^{-}\right\} \geq(3 k-1) / 2$, then $T$ has at least $k$ disjoint cycles. 


\section{Proofs}

Proof of Theorem 1.1. Note that if a bipartite tournament has $r$ disjoint cycles, then it has $r$ disjoint 4-cycles. Suppose that $T$ has exactly $r$ disjoint 4-cycles. Let $\mathcal{C}$ be a family of $r$ disjoint 4-cycles and let $T_{1}$ be the subdigraph induced by $\mathcal{C}$. If $T_{1}=T$, then every vertex $x \in V(T)$ is on a 4-cycle, yielding that $d^{+}(x) \leq 2(r-1)+1=2 r-1$. By hypothesis $d^{+}(x) \geq \delta^{+}(T) \geq 2 k-1$ yielding that $r \geq k$. If $T_{1} \subset T$, let $T_{2}=T-V\left(T_{1}\right)$, clearly $T_{2}$ is an acyclic digraph. Let $v \in V\left(T_{2}\right)$ such that $d_{T_{2}}^{+}(v)=0$. Hence, $N^{+}(v) \subseteq V\left(T_{1}\right)$ and $d^{+}(v) \leq 2 r$. By hypothesis $d^{+}(v) \geq \delta^{+}(T) \geq 2 k-1$ yielding that $r \geq k$.

Proof of Theorem 1.2. Let $k \geq 3$ and let $T=(X, Y)$ be a bipartite tournament with $\delta^{+}(T) \geq 2 k-2$ and $\delta^{-}(T) \geq 1$. By Theorem 1.1, we may assume that $\delta^{+}(T)=2 k-2$, and since $2 k-2>2(k-1)-1$ and $k-1 \geq 2$, it follows by Theorem 1.1 that $T$ has at least $k-1$ disjoint 4 -cycles. Let us denote these cycles by $\left(a_{i}, b_{i}, c_{i}, d_{i}, a_{i}\right)$ for all $1 \leq i \leq k-1$ and let $T_{1}$ be the bipartite tournament induced by these $k-1$ cycles. Let $T_{1}=\left(X_{1}, Y_{1}\right)$ with $X_{1}=\left\{a_{i}, c_{i}: i=1, \ldots, k-1\right\}$ and $Y_{1}=\left\{b_{i}, d_{i}: i=1, \ldots, k-1\right\}$. Let $T_{2}=\left(X_{2}, Y_{2}\right)$ be the bipartite tournament induced by $V(T) \backslash V\left(T_{1}\right)$. Observe that $T_{2}$ is nonempty because by hypothesis the partite sets of $T$ have cardinality at least $2 k$. Clearly, if $T_{2}$ has a cycle we are done. Then we assume that $T_{2}$ is acyclic. In order to prove the existence of $k$ disjoint cycles, we use the vertices of $T_{2}$ and the vertices of one of the cycles $\left(a_{i}, b_{i}, c_{i}, d_{i}, a_{i}\right)$ from $T_{1}$ to construct two new 4-cycles.

Without loss of generality, suppose that $T_{2}$ has a sink $x_{s} \in X_{2}$. Then $Y_{2}=N^{-}\left(x_{s}\right)$ and $N^{+}\left(x_{s}\right)=Y_{1}$ because $d^{+}\left(x_{s}\right) \geq$ $2 k-2=\left|Y_{1}\right|$. Let $x \in X_{1}, y \in Y_{1}$. Since $\left|N^{+}(x) \cap Y_{1}\right|,\left|N^{+}(y) \cap X_{1}\right| \leq 2 k-3$, and $d^{+}(x), d^{+}(y) \geq 2 k-2$ it follows that for all $x \in X_{1}$ and for all $y \in Y_{1}$,

$$
\left|N^{+}(x) \cap Y_{2}\right| \geq 1,\left|N^{+}(y) \cap\left(X_{2}-x_{s}\right)\right| \geq 1 .
$$

Case 1. Suppose $x_{s}^{\prime} \in X_{2}-x_{s}$ is a sink of $T_{2}-x_{s}$. Then $N^{-}\left(x_{s}^{\prime}\right)=Y_{2}$ and $N^{+}\left(x_{s}^{\prime}\right)=Y_{1}$, so that $x_{s}$ and $x_{s}^{\prime}$ are twins in $T$. By (1), we can take $y_{a_{i}} \in N^{+}\left(a_{i}\right) \cap Y_{2}$ and $y_{c_{i}} \in N^{+}\left(c_{i}\right) \cap Y_{2}$. If there is $j \in\{1, \ldots, k-1\}$ such that are $y_{a_{j}} \neq y_{c_{j}}$, then $\left(a_{j}, y_{a_{j}}, x_{s}, d_{j}, a_{j}\right)$ and $\left(c_{j}, y_{c_{j}}, x_{s}^{\prime}, b_{j}, c_{j}\right)$ are two disjoint $C_{4}$ and we are done.

Let us assume that for all $i \in\{1, \ldots, k-1\}$,

$$
N^{+}\left(a_{i}\right) \cap Y_{2}=N^{+}\left(c_{i}\right) \cap Y_{2}=\left\{y_{i}\right\} \text { where } y_{i} \in Y_{2} .
$$

Hence, $N^{+}\left(a_{i}\right)=\left(Y_{1}-d_{i}\right) \cup\left\{y_{i}\right\}, N^{+}\left(c_{i}\right)=\left(Y_{1}-b_{i}\right) \cup\left\{y_{i}\right\}$ for all $i \in\{1, \ldots, k-1\}$. This implies that $N^{+}\left(b_{i}\right) \cap X_{1}=\left\{c_{i}\right\}$ and $N^{+}\left(d_{i}\right) \cap X_{1}=\left\{a_{i}\right\}$ for all $i \in\{1, \ldots, k-1\}$ because $T$ is a bipartite tournament. Thus, $N^{+}\left(b_{i}\right)-c_{i}, N^{+}\left(d_{i}\right)-a_{i} \subseteq X_{2} \backslash\left\{x_{s}, x_{s}^{\prime}\right\}$ for all $i \in\{1, \ldots, k-1\}$.

Let $x_{b_{i}} \in N^{+}\left(b_{i}\right) \cap X_{2}$ and $x_{d_{i}} \in N^{+}\left(d_{i}\right) \cap X_{2}$ (note that both are different from $x_{s}$ and $x_{s}^{\prime}$ ). Since $\left|N^{+}\left(x_{b_{i}}\right) \cap Y_{1}\right| \leq\left|Y_{1}-b_{i}\right|=$ $2 k-3$, there is $y_{b_{i}} \in N^{+}\left(x_{b_{i}}\right) \cap Y_{2}$ and similarly, there is $y_{d_{i}} \in N^{+}\left(x_{d_{i}}\right) \cap Y_{2}$. If $y_{b_{j}} \neq y_{j}$ for some $j \in\{1, \ldots, k-1\}$, then $\left(a_{j}, y_{j}, x_{s}, d_{j}, a_{j}\right)$ and $\left(x_{b_{j}}, y_{b_{j}}, x_{s}^{\prime}, b_{j}, x_{b_{j}}\right)$ are two disjoint $C_{4}$. And if $y_{d_{j}} \neq y_{j}$ for some $j \in\{1, \ldots, k-1\}$, then $\left(c_{j}, y_{j}, x_{s}, b_{j}, c_{j}\right)$ and $\left(x_{d_{j}}, y_{d_{j}}, x_{s}^{\prime}, d_{j}, x_{d_{j}}\right)$ are two disjoint $C_{4}$. In both cases we have $k$ disjoint cycles. Therefore, assume that for all $i \in\{1, \ldots, k-1\}$,

$$
N^{+}\left(x_{b_{i}}\right) \cap Y_{2}=N^{+}\left(x_{d_{i}}\right) \cap Y_{2}=N^{+}\left(a_{i}\right) \cap Y_{2}=N^{+}\left(c_{i}\right) \cap Y_{2}=\left\{y_{i}\right\} .
$$

Since $T_{2}$ is acyclic, it follows that $T_{2}$ has a source. Suppose that $\hat{x} \in X_{2} \backslash\left\{x_{s}, x_{s}^{\prime}\right\}$ is a source of $T_{2}$. Since $d^{-}(\hat{x}) \geq 1$, it follows that there is some $y \in Y_{1}$ such that $y \in N^{-}(\hat{x})$. Then $y=b_{i}$ or $y=d_{i}$, so that $\hat{x} \in N^{+}\left(b_{i}\right) \cup N^{+}\left(d_{i}\right)$, yielding that $\hat{x} \in\left\{x_{b_{i}}, x_{d_{i}}\right\}$, which contradicts (3) because $Y_{2} \subseteq N^{+}(\hat{x})$ and $\left|Y_{2}\right| \geq 2 k-\left|Y_{1}\right|=2 k-2 k+2=2$. Hence, every source of $T_{2}$ is $\hat{y} \in Y_{2}$, implying that $X_{2} \subseteq N^{+}(\hat{y})$. Since $d^{-}(\hat{y}) \geq 1$, it follows that there is some $x \in X_{1}$ such that $x \in N^{-}(\hat{y})$ and $x=a_{i}$ or $x=c_{i}$. By (2), $\hat{y}=y_{i}$, which is a contradiction, because $x_{b_{i}}, x_{d_{i}} \in N^{-}\left(y_{i}\right)$. Thus, in Case 1 , we have $k$ disjoint cycles.

Case 2. Suppose that $y_{s}^{\prime} \in Y_{2}$ is a sink of $T_{2}-x_{s}$. Then $X_{2}-x_{s} \subseteq N^{-}\left(y_{s}^{\prime}\right)$ and let $Z_{s}=N^{-}\left(y_{s}^{\prime}\right) \cap X_{1}$ with $\left|Z_{s}\right| \leq 1$, note that $N^{+}\left(y_{s}^{\prime}\right)=\left(X_{1}-Z_{s}\right) \cup\left\{x_{s}\right\}$, because $d^{+}\left(y_{s}^{\prime}\right) \geq 2 k-2$.

Case 2.1 Suppose that $\left|Z_{s}\right|=1$. Without loss of generality, suppose that $Z_{s}=\left\{a_{1}\right\}$. By (1), let $x_{b_{1}} \in N^{+}\left(b_{1}\right) \cap\left(X_{2}-x_{s}\right)$. Since $\left|N^{+}\left(x_{b_{1}}\right) \cap Y_{1}\right| \leq\left|Y_{1}-b_{1}\right|=2 k-3$, there is $y_{b_{1}} \in N^{+}\left(x_{b_{1}}\right) \cap Y_{2}$. If $y_{s}^{\prime} \neq y_{b_{1}}$, then $\left(y_{s}^{\prime}, c_{1}, d_{1}, a_{1}, y_{s}^{\prime}\right)$ and $\left(x_{s}, b_{1}, x_{b_{1}}, y_{b_{1}}, x_{s}\right)$ are two disjoint $C_{4}$, and we have $k$ disjoint cycles. Therefore, we assume that

$$
y_{b_{1}}=y_{s}^{\prime} \text { and } N^{-}\left(x_{b_{1}}\right)=\left(Y_{2}-y_{s}^{\prime}\right) \cup\left\{b_{1}\right\}, N^{+}\left(x_{b_{1}}\right)=\left\{y_{s}^{\prime}\right\} \cup\left(Y_{1}-b_{1}\right) .
$$

By hypothesis $k \geq 3$, and by (1) we can take $y_{a_{i}} \in N^{+}\left(a_{i}\right) \cap Y_{2}$ and $x_{b_{i}} \in N^{+}\left(b_{i}\right) \cap\left(X_{2}-x_{s}\right)$ for all $i=2, \ldots, k-1$. Hence, by (4), we have $\left(a_{i}, y_{a_{i}}, x_{b_{1}}, d_{i}, a_{i}\right)$ and $\left(x_{b_{i}}, y_{s}^{\prime}, x_{s}, b_{i}, x_{b_{i}}\right)$ are two disjoint $C_{4}$ because $y_{a_{i}} \neq y_{s}^{\prime}$ since $y_{s}^{\prime} \in N^{-}\left(a_{i}\right)$. Hence, we have $k$ disjoint cycles.

Case 2.2 Suppose that $\left|Z_{s}\right|=0$. Then $N^{+}\left(y_{s}^{\prime}\right)=X_{1} \cup\left\{x_{s}\right\}$. If $x_{s}^{\prime \prime} \in X_{2}-x_{s}$ is a sink of $T_{3}=T_{2}-\left\{x_{s}, y_{s}^{\prime}\right\}$, then $\left|N^{-}\left(x_{s}^{\prime \prime}\right) \cap Y_{1}\right| \leq 1$. Since $k \geq 3$ there exists a cycle $\left(a_{i}, b_{i}, c_{i}, d_{i}, a_{i}\right)$ such that $b_{i}, d_{i} \in N^{+}\left(x_{s}^{\prime \prime}\right)$. By (1) there is a vertex $y_{a_{i}} \in N^{+}\left(a_{i}\right) \cap Y_{2}$ and a vertex $x_{b_{i}} \in N^{+}\left(b_{i}\right) \cap X_{2}$. In this case $\left(d_{i}, a_{i}, y_{a_{i}}, x_{s}^{\prime \prime}, d_{i}\right)$ and $\left(b_{i}, x_{b_{i}}, y_{s}^{\prime}, x_{s}, b_{i}\right)$ are two disjoint cycles. Hence, $T$ has $k$ disjoint cycles.

Therefore, we may assume that any sink of $T_{3}$ is a vertex $y_{s}^{\prime \prime}$ of $Y_{2}-y_{s}^{\prime}$. Observe that $y_{s}^{\prime \prime}$ is a sink of $T_{2}-\left\{x_{s}\right\}$, and by Case 2.1 we may assume that $N^{+}\left(y_{s}^{\prime \prime}\right)=X_{1} \cup\left\{x_{s}\right\}$. Let $x_{b_{i}} \in N^{+}\left(b_{i}\right) \cap\left(X_{2}-x_{s}\right)$ and $x_{d_{i}} \in N^{+}\left(d_{i}\right) \cap\left(X_{2}-x_{s}\right)$. Then $\left(a_{i}, b_{i}, x_{b_{i}}, y_{s}^{\prime}, a_{i}\right)$ and $\left(c_{i}, d_{i}, x_{d_{i}}, y_{s}^{\prime \prime}, c_{i}\right)$ are two disjoint cycles. Hence, $T$ has $k$ disjoint cycles.

Therefore we conclude that in either case $T$ must have at least $k$ disjoint cycles and the theorem holds. 
Proof of Corollary 1.1. The girth of a bipartite tournament containing a cycle is $g=4$. Suppose that $k=2$, and let $T$ be a bipartite tournament with $\delta^{+}(T) \geq\lceil 8 / 3\rceil=3$. From Theorem 1.1, it follows that $T$ has at least 2 disjoint cycles. Suppose $k=3$, and let $T$ be a bipartite tournament with $\delta^{+}(T) \geq\lceil 12 / 3\rceil=4=2 \cdot 3-2$. From Theorem 1.2, $T$ has at least 3 disjoint cycles. Analogously, for $k=4, T$ has at least 4 disjoint cycles.

Proof of Theorem 1.3. Let $T=(X, Y)$ be a bipartite tournament with $\delta(T)=\min \left\{\delta^{+}, \delta^{-}\right\} \geq 2$. Thus, $T$ is not acyclic and $T$ has a 4-cycle $C=(a, b, c, d, a)$. Let $T^{\prime}=\left(X^{\prime}, Y^{\prime}\right)$ be the bipartite tournament induced by $V(T) \backslash V(C)$. If $T^{\prime}$ is not acyclic, then we are done. Assume that $T^{\prime}$ is an acyclic bipartite tournament. In order to prove the existence of 2 disjoint cycles, we use the vertices of $T^{\prime}$ and the vertices of $C$ to construct two new 4-cycles. Moreover, $\left|X^{\prime}\right|,\left|Y^{\prime}\right| \geq 2$, because for all $x \in\{a, c\} \cup X^{\prime}$, $d^{-}(x)+d^{+}(x)=|\{b, d\}|+\left|Y^{\prime}\right| \geq 4$; and for all $y \in\{b, d\} \cup Y^{\prime}, d^{-}(y)+d^{+}(y)=|\{a, c\}|+\left|X^{\prime}\right| \geq 4$. Without loss of generality, we may assume that $\hat{x} \in X^{\prime}$ is a source of $T^{\prime}$. Hence, $N^{+}(\hat{x})=Y^{\prime}$ and $N^{-}(\hat{x})=\{b, d\}$. Moreover, $T^{\prime}$ has also a sink, let us distinguish the following cases according to where the sink is placed.

Case 1 . Suppose $T^{\prime}$ has a sink $x_{s} \in X^{\prime}-\hat{x}$. Then $N^{-}\left(x_{s}\right)=Y^{\prime}$ and $N^{+}\left(x_{s}\right)=\{b, d\}$.

If there exists $y_{0} \in Y^{\prime}$ such that the vertices $\left\{c, y_{0}, a\right\}$ induce a path of length 2 in $T$, then $\left(a, b, c, y_{0}, a\right)$ and $\left(\hat{x}, y, x_{s}, d, \hat{x}\right)$, for $y \in Y^{\prime}-y_{0}$ (or $\left(c, d, a, y_{0}, c\right)$ and $\left(\hat{x}, y, x_{s}, b, \hat{x}\right)$, for $\left.y \in Y^{\prime}-y_{0}\right)$ are 2 disjoint 4-cycles in $T$, and we are done. Therefore, assume that

$$
N^{+}(c) \cap N^{-}(a) \cap Y^{\prime}=\emptyset \text { and } N^{-}(c) \cap N^{+}(a) \cap Y^{\prime}=\emptyset .
$$

In this case, $\left|X^{\prime}\right| \geq 3$, else $d^{+}(y)=1$ or $d^{-}(y)=1$ for every $y \in Y^{\prime}$ which is a contradiction. Let us consider the acyclic bipartite tournament $\hat{T}=T^{\prime}-\left\{\hat{x}, x_{s}\right\}$.

Case 1.1. Suppose that $\hat{T}$ has a source $\hat{x}^{\prime} \in X^{\prime} \backslash\left\{\hat{x}, x_{s}\right\}$. Then $N^{+}\left(\hat{x}^{\prime}\right)=Y^{\prime}$ and $N^{-}\left(\hat{x}^{\prime}\right)=\{b, d\}$, that is, $\hat{x}$ and $\hat{x}^{\prime}$ are twins in $T$.

If $x_{s}^{\prime} \in X^{\prime} \backslash\left\{\hat{x}, x_{s}, \hat{x}^{\prime}\right\}$ is a sink of $\hat{T}$, then $N^{-}\left(x_{s}^{\prime}\right)=Y^{\prime}$ and $N^{+}\left(x_{s}^{\prime}\right)=\{b, d\}$, yielding that $\left(\hat{x}, y, x_{s}, d, \hat{x}\right)$ for $y \in Y^{\prime}$ and $\left(\hat{x}^{\prime}, y^{\prime}, x_{s}^{\prime}, b, \hat{x}^{\prime}\right)$ for $y^{\prime} \in Y^{\prime}-y$, are two disjoint 4-cycles, and we are done. Therefore any sink of $\hat{T}$ must be some $y_{s}^{\prime} \in Y^{\prime}$, so that $X^{\prime}-x_{s} \subseteq N^{-}\left(y_{s}^{\prime}\right)$. Let us show that $N^{+}\left(y_{s}^{\prime}\right)=\{a, c\} \cup\left\{x_{s}\right\}$. Indeed, if $a \notin N^{+}\left(y_{s}^{\prime}\right)$, by (5), $c \notin N^{+}\left(y_{s}^{\prime}\right)$, yielding that $d^{+}\left(y_{s}^{\prime}\right) \leq 1$ which is a contradiction. Then $N^{+}\left(y_{s}^{\prime}\right)=\{a, c\} \cup\left\{x_{s}\right\}$. Thus, $\left(\hat{x}, y_{s}^{\prime}, c, d, \hat{x}\right)$ and $\left(\hat{x}^{\prime}, y, x_{s}, b, \hat{x}^{\prime}\right)$ are two disjoint 4-cycles for all $y \in Y^{\prime}-y_{s}^{\prime}$, and we are done.

Case 1.2. Suppose that $\hat{T}$ has a source $\hat{y}^{\prime} \in Y^{\prime}$. Then $N^{+}\left(\hat{y}^{\prime}\right)=X^{\prime}-\hat{x}$ and $N^{-}\left(\hat{y}^{\prime}\right)=\{a, c\} \cup\{\hat{x}\}$ because (5) and $\delta^{-}(T) \geq 2$. If $x_{s}^{\prime} \in X^{\prime} \backslash\left\{\hat{x}, x_{s}\right\}$ is a sink of $\hat{T}$, then $\left(a, \hat{y}^{\prime}, x_{s}^{\prime}, d, a\right)$ and $\left(\hat{x}, y, x_{s}, b, \hat{x}\right)$ for $y \in Y^{\prime}-\hat{y}^{\prime}$, are two disjoint 4-cycles, and we are done. Hence any sink of $\hat{T}$ must be some $y_{s}^{\prime} \in Y^{\prime}-\hat{y}^{\prime}$. Suppose $Y^{\prime}=\left\{\hat{y}^{\prime}, y_{s}^{\prime}\right\}$. Then for every $x \in X^{\prime} \backslash\left\{\hat{x}, x_{s}\right\}$, $\left|N^{+}(x) \cap\{b, d\}\right|=\left|N^{-}(x) \cap\{b, d\}\right|=1$. If $b \in N^{+}(x)$, then $\left(x, b, c, \hat{y}^{\prime}, x\right)$ and $\left(x_{s}, d, \hat{x}, y_{s}^{\prime}, x_{s}\right)$ are two disjoint 4-cycles; and if $d \in N^{+}(x)$, then $\left(x, d, a, \hat{y}^{\prime}, x\right)$ and $\left(x_{s}, b, \hat{x}, y_{s}^{\prime}, x_{s}\right)$ are two disjoint 4-cycles. Hence, we may assume that $\left|Y^{\prime}\right| \geq 3$, then the 4-cycle $\left(a, \hat{y}^{\prime}, x, y_{s}^{\prime}, a\right)$, for $x \in X^{\prime} \backslash\left\{\hat{x}, x_{s}\right\}$, and the 4-cycle $\left(b, \hat{x}, y, x_{s}, b\right)$, for $y \in Y^{\prime} \backslash\left\{\hat{y}^{\prime}, y_{s}^{\prime}\right\}$, are two disjoint cycles, and we are done.

Case 2. Suppose that $y_{s} \in Y^{\prime}$ is a sink of $T^{\prime}$. Then $N^{-}\left(y_{s}\right)=X^{\prime}$ and $N^{+}\left(y_{s}\right)=\{a, c\}$. Consider the bipartite tournament $\hat{T}=T^{\prime}-\left\{\hat{x}, y_{s}\right\}$, which is clearly acyclic.

Case 2.1. Some vertex $\hat{x}^{\prime} \in X^{\prime}-\hat{x}$ is a source of $\hat{T}$. Then $N^{+}\left(\hat{x}^{\prime}\right)=Y^{\prime}$ and $N^{-}\left(\hat{x}^{\prime}\right)=\{b, d\}$.

If $y_{s}^{\prime} \in Y^{\prime}-y_{s}$ is a sink of $\hat{T}$, then it is also a sink of $T^{\prime}$, yielding that $N^{-}\left(y_{s}^{\prime}\right)=X^{\prime}$ and $N^{+}\left(y_{s}^{\prime}\right)=\{a, c\}$. In this case $\left(\hat{x}, y_{s}, a, b, \hat{x}\right)$ and $\left(\hat{x}^{\prime}, y_{s}^{\prime}, c, d, \hat{x}^{\prime}\right)$ are two disjoint 4-cycles in $T$, and we are done. Therefore, any sink of $\hat{T}$ is some $x_{s}^{\prime} \in X^{\prime} \backslash\left\{\hat{x}, \hat{x}^{\prime}\right\}$. Then $Y^{\prime}-y_{s} \subseteq N^{-}\left(x_{s}^{\prime}\right)$ and $\left|N^{+}\left(x_{s}^{\prime}\right) \cap\{b, d\}\right| \geq 1$ since $y_{s} \in N^{+}\left(x_{s}^{\prime}\right)$ and $d^{+}\left(x_{s}^{\prime}\right) \geq 2$. Let $v^{\prime} \in\{b, d\} \cap N^{+}\left(x_{s}^{\prime}\right), v \in\{b, d\}-v^{\prime}$ and $\alpha \in\{a, c\} \cap N^{-}(v)$. Then $\left(\hat{x}, y_{s}, \alpha, v, \hat{x}\right)$ and $\left(\hat{x}^{\prime}, y, x_{s}^{\prime}, v^{\prime}, \hat{x}^{\prime}\right)$ for $y \in Y^{\prime}-y_{s}$, are two disjoint 4-cycles. This gives that $T$ has at least 2 disjoint cycles, and we are done.

Case 2.2. Every source of $\hat{T}$ is some $\hat{y}^{\prime} \in Y^{\prime}-y_{s}$. Then $X^{\prime}-\hat{x} \subseteq N^{+}\left(\hat{y}^{\prime}\right)$ and $\left|N^{-}\left(\hat{y}^{\prime}\right) \cap\{a, c\}\right| \geq 1$ because $\hat{x} \in N^{-}\left(\hat{y}^{\prime}\right)$ and $d^{-}\left(\hat{y}^{\prime}\right) \geq 2$. Hence, there is $t \in N^{-}\left(\hat{y}^{\prime}\right) \cap\{a, c\}$, implying that $\left(t, \hat{y}^{\prime}, x, y_{s}, t\right)$ for all $x \in X^{\prime}-\hat{x}$ is a 4 -cycle in $T$.

If $y_{s}^{\prime} \in Y^{\prime} \backslash\left\{y_{s}, \hat{y}^{\prime}\right\}$ is a sink of $\hat{T}$, then it is also a sink of $T^{\prime}$, yielding that $N^{-}\left(y_{s}^{\prime}\right)=X^{\prime}$ and $N^{+}\left(y_{s}^{\prime}\right)=\{a, c\}$. Then $\left(\hat{x}, y_{s}^{\prime}, z, w, \hat{x}\right)$ where $z \in\{a, c\}-t, w \in\{b, d\}$ and $z w \in A(T)$, is a 4-cycle disjoint with $\left(t, \hat{y}^{\prime}, x, y_{s}, t\right)$ for $x \in X^{\prime}-\hat{x}$. Thus, $T$ has at least 2 disjoint cycles, and we are done.

If $x_{s}^{\prime} \in X^{\prime}-\hat{x}$ is a sink of $\hat{T}$, then $Y^{\prime}-y_{s} \subseteq N^{-}\left(x_{s}^{\prime}\right)$, and $\left|N^{+}\left(x_{s}^{\prime}\right) \cap\{b, d\}\right| \geq 1$ because $y_{s} \in N^{+}\left(x_{s}^{\prime}\right)$ and $d^{+}\left(x_{s}^{\prime}\right) \geq 2$. If $\left|Y^{\prime}\right| \geq 3$, $\left(\hat{x}, y, x_{s}^{\prime}, w, \hat{x}\right)$ for $y \in Y^{\prime} \backslash\left\{y_{s}, \hat{y}^{\prime}\right\}$ and $w \in\{b, d\} \cap N^{+}\left(x_{s}^{\prime}\right)$, is a 4-cycle disjoint with $\left(t, \hat{y}^{\prime}, x, y_{s}, t\right)$ for all $x \in X^{\prime} \backslash\left\{\hat{x}, x_{s}^{\prime}\right\}$, and we are done. Thus, assume that $Y^{\prime}=\left\{y_{s}, \hat{y}^{\prime}\right\}$. If $N^{-}\left(\hat{y}^{\prime}\right) \cap\{a, c\}=\{c\}$, then $N^{+}\left(\hat{y}^{\prime}\right) \cap\{a, c\}=\{a\}$, yielding that $N^{+}(a)=\{b\}$ which is a contradiction. Therefore $N^{-}\left(\hat{y}^{\prime}\right) \cap\{a, c\}=\{a, c\}$. Similarly, if $\left|N^{+}\left(x_{s}^{\prime}\right) \cap\{b, d\}\right|=2$, then $N^{-}\left(x_{s}^{\prime}\right)=\left\{\hat{y}^{\prime}\right\}$ which is a contradiction. If $x_{s}^{\prime} b, d x_{s}^{\prime} \in A(T)$, then $\left(x_{s}^{\prime}, b, c, d, x_{s}^{\prime}\right)$ and $\left(a, \hat{y}^{\prime}, x, y_{s}, a\right)$ are two disjoint $C_{4}$ and we are done. If $b x_{s}^{\prime}, x_{s}^{\prime} d \in A(T)$, then $\left(x_{s}^{\prime}, d, a, b, x_{s}^{\prime}\right)$ and $\left(c, \hat{y}^{\prime}, x, y_{s}, c\right)$ for $x \in X^{\prime} \backslash\left\{\hat{x}, x_{s}^{\prime}\right\}$, are two disjoint $C_{4}$.

Therefore we conclude that $T$ must have at least 2 disjoint cycles.

Proof of Theorem 1.4. If $k=2$ the result holds by Theorem 1.3. Let $k=3,4$, and observe that $\lceil(3 k-1) / 2\rceil=2 k-2$ for these two values. Let $T=(X, Y)$ and note that for all $x \in X, d(x)=d^{-}(x)+d^{+}(x)=|Y| \geq 2(2 k-2)>2 k$; and for all $y \in Y$, $d(y)=d^{-}(y)+d^{+}(y)=|X| \geq 2(2 k-2)>2 k$. Hence, by Theorem 1.2 the theorem holds for $k=3$, 4. Thus, assume $k \geq 5$. We reason by induction on $k$, so assume that the theorem holds for any value less than or equal to $k-1$, that is, $T$ has $k-1$ disjoint cycles by the induction hypothesis. Let us denote these cycles by $\left(a_{i}, b_{i}, c_{i}, d_{i}, a_{i}\right)$ for all $i=1,2, \ldots, k-1$ and let 
$T_{1}=\left(X_{1}, Y_{1}\right), T_{2}=\left(X_{2}, Y_{2}\right)$, and $T=\left(X_{1} \cup X_{2}, Y_{1} \cup Y_{2}\right)$ be the same as in Theorem 1.2. Without loss of generality, assume that $\hat{x} \in X_{2}$ is a source of $T_{2}$, that is, $Y_{2} \subseteq N^{+}(\hat{x})$. Let $\hat{V}=N^{+}(\hat{x}) \cap Y_{1}$, then $Y_{1} \backslash \hat{V}=N^{-}(\hat{x})$ and $|\hat{V}| \leq(k-3) / 2$, because $\left|Y_{1}\right|=2 k-2$ and $\delta(T) \geq(3 k-1) / 2$. Observe that $\left|X_{2}\right|>2$, because if $\left|X_{2}\right|=2$, then $|\hat{V}| \geq(3 k-1) / 2-2>(k-3) / 2$, which is a contradiction. As in the proof of Theorem 1.2, we will find two disjoint $C_{4}$ using vertices of just one cycle $\left(a_{i}, b_{i}, c_{i}, d_{i}, a_{i}\right)$ and vertices of $X_{2} \cup Y_{2}$. Hence, $T$ will have $k$ disjoint cycles. Since $T_{2}$ is acyclic, it has also a sink. Let us distinguish the following cases according the location of a sink of $T_{2}$.

Case 1. $T_{2}$ has a sink $x_{s} \in X_{2}-\hat{x}$. Then $Y_{2} \subseteq N^{-}\left(x_{s}\right)$ and let $V_{s}=N^{-}\left(x_{s}\right) \cap Y_{1}$. Therefore $Y_{1} \backslash V_{s}=N^{+}\left(x_{s}\right)$ and $\left|V_{s}\right| \leq(k-3) / 2$, because $\left|Y_{1}\right|=2 k-2$ and $\delta(T) \geq(3 k-1) / 2$. Let us consider the acyclic bipartite tournament $T_{3}=T_{2}-\left\{\hat{x}, x_{s}\right\}$.

Case 1.1. $T_{3}$ has a source $\hat{x}^{\prime} \in X_{2} \backslash\left\{\hat{x}, x_{s}\right\}$. Then $Y_{2} \subseteq N^{+}\left(\hat{x}^{\prime}\right)$ and let $\hat{V}^{\prime}=N^{+}\left(\hat{x}^{\prime}\right) \cap Y_{1}$. Therefore $Y_{1} \backslash \hat{V}^{\prime}=N^{-}\left(\hat{x}^{\prime}\right)$ and $\left|\hat{V}^{\prime}\right| \leq(k-3) / 2$.

Case 1.1.1. $x_{s}^{\prime} \in X_{2} \backslash\left\{\hat{x}, x_{s}, \hat{x}^{\prime}\right\}$ is a sink of $T_{3}$. Then $Y_{2} \subseteq N^{-}\left(x_{s}^{\prime}\right)$ and $Y_{1} \backslash V_{s}^{\prime}=N^{+}\left(x_{s}^{\prime}\right)$ where $V_{s}^{\prime}=N^{-}\left(x_{s}^{\prime}\right) \cap Y_{1}$ and $\left|V_{s}^{\prime}\right| \leq(k-3) / 2$. If there exists $i \in\{1, \ldots, k-1\}$ such that $\left|\left\{b_{i}, d_{i}\right\} \cap\left(\hat{V} \cup \hat{V}^{\prime} \cup V_{s} \cup V_{s}^{\prime}\right)\right|=0$, then $\left(\hat{x}, y, x_{s}, d_{i}, \hat{x}\right)$ for $y \in Y_{2}$, and $\left(\hat{x}^{\prime}, y^{\prime}, x_{s}^{\prime}, b_{i}, \hat{x}^{\prime}\right)$ for $y^{\prime} \in Y_{2}-y$, are two disjoint 4-cycles and we are done. Thus, we assume for all $i \in\{1, \ldots, k-1\}$ that $\left|\left\{b_{i}, d_{i}\right\} \cap\left(\hat{V} \cup \hat{V}^{\prime} \cup V_{s} \cup V_{s}^{\prime}\right)\right| \geq 1$. For $h=1$, 2 let $R_{h}=\left\{i \in\{1, \ldots, k-1\}:\left|\left\{b_{i}, d_{i}\right\} \cap\left(\hat{V} \cup \hat{V}^{\prime} \cup V_{s} \cup V_{s}^{\prime}\right)\right|=h\right\}$. We have

$$
\left|\hat{V} \cup \hat{V}^{\prime} \cup V_{s} \cup V_{s}^{\prime}\right|=2\left|R_{2}\right|+\left|R_{1}\right|=\left|R_{2}\right|+k-1 \text {. }
$$

Moreover, let $I=\left(V_{s} \cup \hat{V}\right) \cap\left(V_{s}^{\prime} \cup \hat{V}^{\prime}\right)$, then $\left|\hat{V} \cup \hat{V}^{\prime} \cup V_{s} \cup V_{s}^{\prime}\right| \leq 2(k-3)-|I|$, which implies that $\left|R_{2}\right| \leq k-5-|I|$. Therefore, $\left|R_{1}\right|=k-1-\left|R_{2}\right| \geq k-1-(k-5-|I|)=4+|I|$. Hence, there exists $i \in R_{1}$ such that $\left|\left\{b_{i}, d_{i}\right\} \cap I\right|=0$. Without loss of generality, suppose $b_{i} \notin \hat{V} \cup \hat{V}^{\prime} \cup V_{s} \cup V_{s}^{\prime}$. As $d_{i} \notin I$ then $d_{i} \notin V_{s} \cup \hat{V}$ or $d_{i} \notin V_{s}^{\prime} \cup \hat{V}^{\prime}$. Without loss of generality, suppose that $d_{i} \notin V_{s} \cup \hat{V}$, then $\left(\hat{x}, y, x_{s}, d_{i}, \hat{x}\right)$ for $y \in Y_{2}$, and $\left(\hat{x}^{\prime}, y^{\prime}, x_{s}^{\prime}, b_{i}, \hat{x}^{\prime}\right)$ for $y^{\prime} \in Y_{2}-y$, are two disjoint 4-cycles and we are done.

Case 1.1.2. $y_{s}^{\prime} \in Y_{2}$ is a sink of $T_{3}$. Thus, $X_{2}-x_{s} \subseteq N^{-}\left(y_{s}^{\prime}\right)$, and $N^{+}\left(y_{s}^{\prime}\right)=\left(X_{1} \backslash Z_{s}^{\prime}\right) \cup\left\{x_{s}\right\}$ where $Z_{s}^{\prime}=N^{-}\left(y_{s}^{\prime}\right) \cap X_{1}$ with $\left|Z_{s}^{\prime}\right| \leq(k-1) / 2$ because $\delta(T) \geq(3 k-1) / 2$. Let $I=\left(V_{s} \cup \hat{V}\right) \cap \hat{V}^{\prime}$ and $R=Y_{1} \backslash\left(\hat{V} \cup \hat{V}^{\prime} \cup V_{s}\right)$. For $h=1$, 2, let $R_{h}=\left\{j \in\{1, \ldots, k-1\}:\left|\left\{b_{j}, d_{j}\right\} \cap R\right|=h\right\}$ and $L_{h}=\left\{j \in\{1, \ldots, k-1\}:\left|\left\{a_{j}, c_{j}\right\} \cap Z_{s}^{\prime}\right|=h\right\}$. Then $2\left|R_{2}\right|+\left|R_{1}\right|=|R|$ and $2\left|L_{2}\right|+\left|L_{1}\right|=\left|Z_{s}^{\prime}\right|$. Suppose that there is $j \in R_{2}$ such that $\left|\left\{a_{j}, c_{j}\right\} \cap Z_{s}^{\prime}\right| \leq 1$. Without loss of generality, suppose that $a_{j} \notin Z_{s}^{\prime}$, then $\left(\hat{x}^{\prime}, y_{s}^{\prime}, a_{j}, b_{j}, \hat{x}^{\prime}\right)$, and $\left(\hat{x}, y, x_{s}, d_{j}, \hat{x}\right)$ for $y \in Y_{2}-y_{s}^{\prime}$ are two disjoint $C_{4}$, and we are done. Therefore we suppose that for all $j \in R_{2},\left|\left\{a_{j}, c_{j}\right\} \cap Z_{s}^{\prime}\right|=2$, that is,

$$
\left|R_{2}\right| \leq\left|L_{2}\right| \text {. }
$$

Since $\left|Y_{1}\right|=\left|\hat{V} \cup \hat{V}^{\prime} \cup V_{s} \cup R\right| \leq 3(k-3) / 2-|I|+|R|$, and $\left|Y_{1}\right|=2 k-2$, it follows that $|R| \geq(k+5) / 2+|I|$, and by (6), $(k+5) / 2+|I| \leq|R|=2\left|R_{2}\right|+\left|R_{1}\right| \leq 2\left|L_{2}\right|+\left|R_{1}\right|$. Let $W=\left\{j \in R_{1} \backslash L_{1}:\left|\left\{b_{j}, d_{j}\right\} \cap I\right|=0\right\}$. If $W=\emptyset$, then $\left|R_{1} \backslash L_{1}\right| \leq|I|$ yielding that $(k+5) / 2+|I| \leq 2\left|L_{2}\right|+\left|R_{1}\right| \leq 2\left|L_{2}\right|+\left|L_{1}\right|+|I|=\left|Z_{s}^{\prime}\right|+|I| \leq(k-1) / 2+|I|$, which is a contradiction. Therefore $W \neq \emptyset$. Suppose that $W \subset L_{2}$. Then $|W|+\left|R_{2}\right| \leq\left|L_{2}\right|$ because $W \cap R_{2}=\emptyset$ by definition of $W$, and by (6). As $|W|=\left|R_{1}\right|-\left|L_{1}\right|-|I|$ we have $\left|R_{2}\right|+\left|R_{1}\right| \leq\left|L_{2}\right|+\left|L_{1}\right|+|I|$. Adding $\left|R_{2}\right|$ on both sides of this inequality we have $|R| \leq\left|R_{2}\right|+\left|L_{2}\right|+\left|L_{1}\right|+|I| \leq 2\left|L_{2}\right|+\left|L_{1}\right|+|I|=\left|Z_{s}^{\prime}\right|+|I| \leq(k-1) / 2+|I|$, which is a contradiction because $|R| \geq(k+5) / 2+|I|$. It follows that there exists $\ell \in W \backslash L_{2}$, that is, $\left|Z_{s}^{\prime} \cap\left\{a_{\ell}, c_{\ell}\right\}\right|=0,\left|\left\{b_{\ell}, d_{\ell}\right\} \cap R\right|=1$ and $\left|\left\{b_{\ell}, d_{\ell}\right\} \cap I\right|=0$.

Without loss of generality, suppose $b_{\ell} \in R$ and $d_{\ell} \notin R$. Since $d_{\ell} \notin I$ we have $d_{\ell} \notin V_{s} \cup \hat{V}$ or $d_{\ell} \notin \hat{V}^{\prime}$. Thus, if $d_{\ell} \notin V_{s} \cup \hat{V}$, then $\left(\hat{x}^{\prime}, y_{s}^{\prime}, a_{\ell}, b_{\ell}, \hat{x}^{\prime}\right)$, and $\left(\hat{x}, y, x_{s}, d_{\ell}, \hat{x}\right)$ for $y \in Y_{2}-y_{s}^{\prime}$ are two disjoint $C_{4}$, and we are done. If $d_{\ell} \notin \hat{V}^{\prime}$, then $\left(\hat{x}, y, x_{s}, b_{\ell}, \hat{x}\right)$ and $\left(\hat{x}^{\prime}, y_{s}^{\prime}, c_{\ell}, d_{\ell}, \hat{x}^{\prime}\right)$ for $y \in Y_{2}-y_{s}^{\prime}$ are two disjoint $C_{4}$, and we are done.

Case 1.2. Any source of $T_{3}$ is some $\hat{y}^{\prime} \in Y_{2}$. Then $X_{2}-\hat{x} \subseteq N^{+}\left(\hat{y}^{\prime}\right)$ and let $\hat{Z}^{\prime}=X_{1} \cap N^{+}\left(\hat{y}^{\prime}\right)$ with $\left|\hat{Z}^{\prime}\right| \leq(k-1) / 2$ such that $\left(X_{1} \backslash \hat{Z}^{\prime}\right) \cup\{\hat{x}\}=N^{-}\left(\hat{y}^{\prime}\right)$, because $\delta(T) \geq(3 k-1) / 2$. Observe that $\left|Y_{2}\right|>2$ because otherwise $\left|\hat{Z}^{\prime}\right| \geq(3 k-1) / 2-2$ which is a contradiction.

Case 1.2.1. $x_{s}^{\prime} \in X_{2} \backslash\left\{\hat{x}, x_{s}\right\}$ is a sink of $T_{3}$. This case is the same as Case 1.1.2. by considering the converse digraph of $T$.

Case 1.2.2. $y_{s}^{\prime} \in Y_{2}-\hat{y}^{\prime}$ is a sink of $T_{3}$. Thus $X_{2}-x_{s} \subseteq N^{-}\left(y_{s}^{\prime}\right)$ and $N^{+}\left(y_{s}^{\prime}\right)=\left(X_{1} \backslash Z_{s}^{\prime}\right) \cup\left\{x_{s}\right\}$ where $Z_{s}^{\prime}=N^{-}\left(y_{s}^{\prime}\right) \cap X_{1}$ with $\left|Z_{s}^{\prime}\right| \leq(k-1) / 2$. For $h=0,1,2$, let $L_{h}=\left\{i \in\{1, \ldots, k-1\}:\left|\left\{a_{i}, c_{i}\right\} \cap\left(\hat{Z}^{\prime} \cup Z_{s}^{\prime}\right)\right|=h\right\}$ and $R_{h}=\{i \in\{1, \ldots, k-1\}$ : $\left.\left|\left\{b_{i}, d_{i}\right\} \cap\left(\hat{V} \cup V_{s}\right)\right|=h\right\}$. If there is $i \in\left(L_{0} \cup L_{1}\right) \cap\left(R_{0} \cup R_{1}\right)$, then without loss of generality we may assume that $a_{i} \notin \hat{Z}^{\prime} \cup Z_{s}^{\prime}$ and $b_{i} \notin \hat{V} \cup V_{s}$. Hence, $\left(\hat{y}^{\prime}, x, y_{s}^{\prime}, a_{i}, \hat{y}^{\prime}\right)$, for $x \in X_{2} \backslash\left\{\hat{x}, x_{s}\right\}$, and $\left(\hat{x}, y, x_{s}, b_{i}, \hat{x}\right)$, for $y \in Y_{2} \backslash\left\{\hat{y}^{\prime}, y_{s}^{\prime}\right\}$, are two disjoint cycles, and we are done. Thus, we must suppose that $\left(L_{0} \cup L_{1}\right) \cap\left(R_{0} \cup R_{1}\right)=\emptyset$ or equivalently, $L_{0} \cup L_{1} \subseteq R_{2}$ and $R_{0} \cup R_{1} \subseteq L_{2}$. Since $\left|\hat{Z}^{\prime} \cup Z_{s}^{\prime}\right| \leq k-1$ it follows that $\left|X_{1} \backslash\left(\hat{Z}^{\prime} \cup Z_{s}^{\prime}\right)\right|=2\left|L_{0}\right|+\left|L_{1}\right|=2 k-2-\left|\hat{Z}^{\prime} \cup Z_{s}^{\prime}\right| \geq k-1=\left|L_{0}\right|+\left|L_{1}\right|+\left|L_{2}\right|$ yielding that $\left|L_{0}\right| \geq\left|L_{2}\right|$ and so $\left|L_{0}\right|+\left|L_{1}\right| \geq(k-1) / 2,\left|L_{2}\right| \leq(k-1) / 2$, and $\left|R_{0}\right|+\left|R_{1}\right| \leq(k-1) / 2$ because $R_{0} \cup R_{1} \subseteq L_{2}$. Furthermore, since $\left|\hat{V} \cup V_{s}\right| \leq k-3$, it follows that $\left|Y_{1} \backslash\left(\hat{V} \cup V_{s}\right)\right|=2\left|R_{0}\right|+\left|R_{1}\right|=2 k-2-\left|\hat{V} \cup V_{s}\right| \geq k+1=\left|R_{0}\right|+\left|R_{1}\right|+\left|R_{2}\right|+2$, yielding $\left|R_{0}\right|+\left|R_{1}\right| \geq(k-1) / 2$, and therefore $\left|R_{0}\right|+\left|R_{1}\right|=(k-1) / 2$. Hence, $2\left|R_{0}\right|+\left|R_{1}\right|=\left|R_{0}\right|+(k-1) / 2 \geq k+1$, and so $\left|R_{0}\right| \geq(k+1) / 2$, which is a contradiction.

Case 2. $T_{2}$ has a sink $y_{s} \in Y_{2}$. Then $X_{2} \subseteq N^{-}\left(y_{s}\right)$ and let $Z_{s}=X_{1} \cap N^{-}\left(y_{s}\right)$ with $\left|Z_{s}\right| \leq(k-3) / 2$ such that $N^{+}\left(y_{s}\right)=X_{1} \backslash Z_{s}$. Let us consider the bipartite tournament $T_{3}=T_{2}-\left\{\hat{x}, y_{s}\right\}$ which is clearly acyclic.

Case 2.1. Some vertex $\hat{x}^{\prime} \in X_{2}-\hat{x}$ is a source of $T_{3}$. Then $Y_{2} \subseteq N^{+}\left(\hat{x}^{\prime}\right)$ and $N^{-}\left(\hat{x}^{\prime}\right)=Y_{1} \backslash \hat{V}^{\prime}$ where $\hat{V}^{\prime}=N^{+}\left(\hat{x}^{\prime}\right) \cap Y_{1}$ with $\left|\hat{V}^{\prime}\right| \leq(k-3) / 2$.

Case 2.1.1. If some $y_{s}^{\prime} \in Y_{2}-y_{s}$ is a sink of $T_{3}$. Then $X_{2} \subseteq N^{-}\left(y_{s}^{\prime}\right)$ and $N^{+}\left(y_{s}^{\prime}\right)=X_{1} \backslash Z_{s}^{\prime}$ where $Z_{s}^{\prime}=N^{-}\left(y_{s}^{\prime}\right) \cap X_{1}$ with $\left|Z_{s}^{\prime}\right| \leq(k-3) / 2$. For $h=0,1,2$, let $L_{h}=\left\{i \in\{1, \ldots, k-1\}:\left|\left\{a_{i}, c_{i}\right\} \cap\left(Z_{s} \cup Z_{s}^{\prime}\right)\right|=h\right\}$. Then $2\left|L_{0}\right|+\left|L_{1}\right|=\left|X_{1} \backslash\left(Z_{s} \cup Z_{s}^{\prime}\right)\right| \geq$ $2 k-2-\left(k-3-\left|Z_{s} \cap Z_{s}^{\prime}\right|\right)=k+1+\left|Z_{s} \cap Z_{s}^{\prime}\right|$. 
Suppose that there is $i \in L_{0}$, that is $\left|\left\{a_{i}, c_{i}\right\} \cap\left(Z_{s} \cup Z_{s}^{\prime}\right)\right|=0$, such that $\left|\left\{b_{i}, d_{i}\right\} \cap\left(\hat{V} \cap \hat{V}^{\prime}\right)\right|=0$. Without loss of generality, suppose that $b_{i} \notin \hat{V}$ and $d_{i} \notin \hat{V}^{\prime}$. Then $\left(\hat{x}^{\prime}, y_{s}, c_{i}, d_{i}, \hat{x}^{\prime}\right)$ and $\left(\hat{x}, y_{s}^{\prime}, a_{i}, b_{i}, \hat{x}\right)$ are disjoint 4-cycles in $T$ and we are done. Therefore we assume that for all $i \in L_{0},\left|\left\{b_{i}, d_{i}\right\} \cap\left(\hat{V} \cap \hat{V}^{\prime}\right)\right| \geq 1$ yielding that $\left|L_{0}\right| \leq\left|\hat{V} \cap \hat{V}^{\prime}\right| \leq(k-3) / 2$. Therefore $\left|L_{1}\right|+\left|L_{0}\right| \geq k+1+\left|Z_{s} \cap Z_{s}^{\prime}\right|-\left|L_{0}\right| \geq(k+5) / 2+\left|Z_{s} \cap Z_{s}^{\prime}\right|$. Hence, there is $i \in L_{0} \cup L_{1}$ (i.e. $\left.\left|\left\{a_{i}, c_{i}\right\} \cap\left(Z_{s} \cup Z_{s}^{\prime}\right)\right| \leq 1\right)$ such that $\left|\left\{a_{i}, c_{i}\right\} \cap\left(Z_{s} \cap Z_{s}^{\prime}\right)\right|=0$, and $\left|\left\{b_{i}, d_{i}\right\} \cap\left(\hat{V} \cap \hat{V}^{\prime}\right)\right|=0$ because $\left|\hat{V} \cap \hat{V}^{\prime}\right| \leq(k-3) / 2$. Without loss of generality, suppose that $a_{i} \notin Z_{s} \cup Z_{s}^{\prime}$ and $c_{i} \notin Z_{s}$. Then if $b_{i} \notin \hat{V}$ and $d_{i} \notin \hat{V}^{\prime}$, then $\left(\hat{x}^{\prime}, y_{s}, c_{i}, d_{i}, \hat{x}^{\prime}\right)$ and $\left(\hat{x}, y_{s}^{\prime}, a_{i}, b_{i}, \hat{x}\right)$ are disjoint 4-cycles in $T$ and we are done. If $b_{i} \notin \hat{V}^{\prime}$ and $d_{i} \notin \hat{V}$, then $\left(\hat{x}, y_{s}, c_{i}, d_{i}, \hat{x}\right)$ and $\left(\hat{x}^{\prime}, y_{s}^{\prime}, a_{i}, b_{i}, \hat{x}^{\prime}\right)$ are disjoint 4-cycles in $T$. Hence, we are done.

Case 2.1.2. Any sink of $T_{3}$ is $x_{s}^{\prime} \in X_{2} \backslash\left\{\hat{x}, \hat{x}^{\prime}\right\}$. Thus, $Y_{2}-y_{s} \subset N^{-}\left(x_{s}^{\prime}\right)$ and let $V_{s}^{\prime}=N^{-}\left(x_{s}^{\prime}\right) \cap Y_{1}$ with $\left|V_{s}^{\prime}\right| \leq(k-1) / 2$ such that $\left(Y_{1} \backslash V_{s}^{\prime}\right) \cup\left\{y_{s}\right\}=N^{+}\left(x_{s}^{\prime}\right)$. (Observe that this case is similar to Case 1.1 .2 but now $\left|Z_{s}\right| \leq(k-3) / 2$ and $\left|V_{s}^{\prime}\right| \leq(k-1) / 2$ ). Let $I=\hat{V} \cap\left(\hat{V}^{\prime} \cup V_{s}^{\prime}\right)$ and $R=Y_{1} \backslash\left(\hat{V} \cup \hat{V}^{\prime} \cup V_{s}^{\prime}\right)$. For $h=1,2$, let $R_{h}=\left\{j \in\{1, \ldots, k-1\}:\left|\left\{b_{j}, d_{j}\right\} \cap R\right|=h\right\}$ and $L_{h}=\left\{j \in\{1, \ldots, k-1\}:\left|\left\{a_{j}, c_{j}\right\} \cap Z_{s}^{\prime}\right|=h\right\}$. Then $2\left|R_{2}\right|+\left|R_{1}\right|=|R|$ and $2\left|L_{2}\right|+\left|L_{1}\right|=\left|Z_{s}\right|$. Suppose that there is $j \in R_{2}$ such that $\left|\left\{a_{j}, c_{j}\right\} \cap Z_{s}\right| \leq 1$. Without loss of generality, suppose that $a_{j} \notin Z_{s}$, then $\left(\hat{x}^{\prime}, y_{s}, a_{j}, b_{j}, \hat{x}^{\prime}\right)$, and $\left(\hat{x}, y, x_{s}^{\prime}, d_{j}, \hat{x}\right)$ for $y \in Y_{2}-y_{s}$ are two disjoint $C_{4}$, and we are done. Therefore we suppose that for all $j \in R_{2},\left|\left\{a_{j}, c_{j}\right\} \cap Z_{s}\right|=2$, that is,

$$
\left|R_{2}\right| \leq\left|L_{2}\right| \text {. }
$$

Since $\left|Y_{1}\right|=\left|\hat{V} \cup \hat{V}^{\prime} \cup V_{s}^{\prime} \cup R\right| \leq(3 k-7) / 2-|I|+|R|$, and $\left|Y_{1}\right|=2 k-2$ it follows that $|R| \geq(k+3) / 2+|I|$ and by (7), $(k+3) / 2+|I| \leq|R|=2\left|R_{2}\right|+\left|R_{1}\right| \leq 2\left|L_{2}\right|+\left|R_{1}\right|$. Let $W=\left\{j \in R_{1} \backslash L_{1}:\left|\left\{b_{j}, d_{j}\right\} \cap I\right|=0\right\}$. If $W=\emptyset$, then $\left|R_{1} \backslash L_{1}\right| \leq|I|$ yielding that $(k+3) / 2+|I| \leq 2\left|L_{2}\right|+\left|R_{1}\right| \leq 2\left|L_{2}\right|+\left|L_{1}\right|+|I|=\left|Z_{s}^{\prime}\right|+|I| \leq(k-1) / 2+|I|$, which is a contradiction. Therefore $W \neq \emptyset$. If $W \subset L_{2}$, then $|W|+\left|R_{2}\right| \leq\left|L_{2}\right|$ because $W \cap R_{2}=\emptyset$ by definition of $W$, and by (7). As $|W|=\left|R_{1}\right|-\left|L_{1}\right|-|I|$ we have $\left|R_{2}\right|+\left|R_{1}\right| \leq\left|L_{2}\right|+\left|L_{1}\right|+|I|$. Adding $\left|R_{2}\right|$ to both sides of the inequality we have $|R| \leq\left|R_{2}\right|+\left|L_{2}\right|+\left|L_{1}\right|+|I| \leq 2\left|L_{2}\right|+\left|L_{1}\right|+|I|=\left|Z_{s}\right|+|I| \leq(k-3) / 2+|I|$, which is a contradiction because $|R| \geq(k+3) / 2+|I|$. It follows that there exists $\ell \in W \backslash L_{2}$, that is, $\left|Z_{s} \cap\left\{a_{\ell}, c_{\ell}\right\}\right|=0,\left|\left\{b_{\ell}, d_{\ell}\right\} \cap R\right|=1$ and $\left|\left\{b_{\ell}, d_{\ell}\right\} \cap I\right|=0$. Without loss of generality, suppose $b_{\ell} \in R$. Since $d_{\ell} \notin I$ we have $d_{\ell} \notin V_{s}^{\prime} \cup \hat{V}^{\prime}$ or $d_{\ell} \notin \hat{V}$. Thus, if $d_{\ell} \notin V_{s}^{\prime} \cup \hat{V}^{\prime}$, then $\left(\hat{x}, y_{s}, a_{\ell}, b_{\ell}, \hat{x}\right)$, and $\left(\hat{x}^{\prime}, y, x_{s}^{\prime}, d_{\ell}, \hat{x}^{\prime}\right)$ for $y \in Y_{2}-y_{s}$ are two disjoint $C_{4}$, and we are done. If $d_{\ell} \notin \hat{V}$, then $\left(\hat{x}^{\prime}, y, x_{s}^{\prime}, b_{\ell}, \hat{x}^{\prime}\right)$ and $\left(\hat{x}, y_{s}, c_{\ell}, d_{\ell}, \hat{x}\right)$ for $y \in Y_{2}-y_{s}$ are two disjoint $C_{4}$, and we are done.

Case 2.2. Every source of $T_{3}$ is a vertex $\hat{y}^{\prime} \in Y_{2}-y_{s}$. Therefore, $X_{2}-\hat{x} \subset N^{+}\left(\hat{y}^{\prime}\right)$ and $N^{-}\left(\hat{y}^{\prime}\right)=\left(X_{1} \backslash \hat{Z}^{\prime}\right) \cup\{\hat{x}\}$ where $\hat{Z}^{\prime}=N^{+}\left(\hat{y}^{\prime}\right) \cap X_{1}$ with $\left|\hat{Z}^{\prime}\right| \leq(k-1) / 2$. Observe that $\left|Y_{2}\right|>2$.

Case 2.2.1. Some $y_{s}^{\prime} \in Y_{2} \backslash\left\{y_{s}, \hat{y}^{\prime}\right\}$ is a sink of $T_{3}$. This case is the same as Case 2.1.2. by considering the converse digraph of $T$.

Case 2.2.2. Any sink of $T_{3}$ is a vertex $x_{s}^{\prime} \in X_{2}-\hat{x}$. Then $Y_{2}-y_{s} \subset N^{-}\left(x_{s}^{\prime}\right)$, and let $V_{s}^{\prime}=N^{-}\left(x_{s}^{\prime}\right) \cap Y_{1}$ with $\left|V_{s}^{\prime}\right| \leq(k-1) / 2$ such that $N^{+}\left(x_{s}^{\prime}\right)=\left(Y_{1} \backslash V_{s}\right) \cup\left\{y_{s}\right\}$. Since $\left|\hat{Z}^{\prime} \cup Z_{s}\right| \leq k-2$ and $\left|\hat{V} \cup V_{s}^{\prime}\right| \leq k-2,\left|X_{1} \backslash\left(\hat{Z}^{\prime} \cup Z_{s}\right)\right| \geq 2 k-2-(k-2)=k$ and $\left|Y_{1} \backslash\left(\hat{V} \cup V_{s}^{\prime}\right)\right| \geq k$. Hence, there exists $\ell \in\{1, \ldots, k-1\}$, such that $\left|\left(\hat{Z}^{\prime} \cup Z_{s}\right) \cap\left\{a_{\ell}, c_{\ell}\right\}\right| \leq 1$ and $\left|\left(\hat{V} \cup V_{s}^{\prime}\right) \cap\left\{b_{\ell}, d_{\ell}\right\}\right| \leq 1$. Without loss of generality, suppose that $a_{\ell} \notin \hat{Z}^{\prime} \cup Z_{s}$ and $d_{\ell} \notin \hat{V} \cup V_{s}^{\prime}$. Then $\left(\hat{x}, y, x_{s}^{\prime}, d_{\ell}, \hat{x}\right)$ for $y \in Y_{2} \backslash\left\{y_{s}, \hat{y}^{\prime}\right\}$, is a $C_{4}$ disjoint with $\left(\hat{y}, x, y_{s}, a_{\ell}, \hat{y}\right)$ for all $x \in X_{2} \backslash\left\{\hat{x}, x_{s}^{\prime}\right\}$, and we are done.

Therefore, we conclude that $T$ must have at least $k$ disjoint cycles.

\section{Acknowledgments}

This work was done while the first author visited Departamento de Matemáticas Aplicadas y Sistemas, UAM Cuajimalpa, Ciudad de México, México. The hospitality and financial support is gratefully acknowledged. This research was supported by the Ministry of "Economía y Competitividad", Spain, and the European Regional Development Fund (ERDF) under project MTM2014-60127-P. The second author's research was supported by CONACyT-México, under project CB-222104.

\section{References}

[1] N. Alon, Disjoint directed cycles, J. Combin. Theory Ser. B 68 (1996) 167-178.

[2] Y. Bai, B. Li, H. Li, Vertex-disjoint cycles in bipartite tournaments, Discrete Math. 338 (2015) 1307-1309.

[3] J. Bang-Jensen, S. Bessy, S. Thomassé, Disjoint 3-cycles in torunaments: A proof of the Bermond-Thomassen conjecture for tournaments, J. Graph Theory 75 (2014) 284-302.

[4] J. Bang-Jensen, G. Gutin, Digraphs: Theory, Algorithms and Applications, second ed., Springer-Verlag, London, 2009.

[5] J.C. Bermond, C. Thomassen, Cycles in digraphs-a survey, J. Graph Theory 5 (1) (1981) 1-43.

[6] S. Bessy, N. Lichiardopol, J.S. Sereni, Two proofs of the Bermond-Thomassen conjecture for tournaments with bounded minimum in-degree, Discrete Math. 310 (2010) 557-560.

[7] N. Lichiardopol, A. Pór, J.S. Sereni, A step towards the Bermond-Thomassen conjecture about disjoint cycles in digraphs, SIAM J. Discrete Math. 23 (2009) 979-992.

[8] C. Thomassen, Disjoint cycles in digraphs, Combinatorica 2 (3-4) (1983) 393-396. 